\title{
Projection-Slice Theorem as a Tool for Mathematical Representation of Diffraction
}

\author{
Levent Onural, Senior Member, IEEE
}

\begin{abstract}
Although the impulse (Dirac delta) function has been widely used as a tool in signal processing, its more complicated counterpart, the impulse function over higher dimensional manifolds in $\mathbb{R}^{N}$, did not get such a widespread utilization. Based on carefully made definitions of such functions, it is shown that many higher dimensional signal processing problems can be better formulated, yielding more insight and flexibility, using these tools. The well-known projection-slice theorem is revisited using these impulse functions. As a demonstration of the utility of the projection-slice formulation using impulse functions over hyperplanes, the scalar optical diffraction is reformulated in a more general context.
\end{abstract}

Index Terms-Curve impulses, diffraction, distributions, generalized functions, impulse functions, projection-slice theorem, radon transform, surface impulses.

\section{INTRODUCTION}

A DEFINITION of impulse functions over a manifold in $N$-dimensional space is given in [1] together with many of its mathematical properties. A preferred definition of these functions is presented in [2] as

$$
\begin{aligned}
\left\langle\delta_{S}(\mathbf{x}), f(\mathbf{x})\right\rangle & =\int_{\mathbb{R}^{N}} \delta_{S}(\mathbf{x}) f(\mathbf{x}) d \mathbf{x} \\
& =\int_{S} f(\mathbf{x}) d S
\end{aligned}
$$

together with some related properties. These functions represent concentration (of mass) over the given manifold. The difference between the two definitions above is in the distribution of the concentrated mass along the manifold. In other words, the definition given in [2] yields uniform mass per unit geometry of the manifold, i.e., uniform mass per unit length of a curve, per unit area of a surface, etc., in $\mathbb{R}^{N}$. Such a definition is more convenient in many engineering applications and easily extends the definition to manifolds that are not smooth or cannot be easily expressed analytically.

As in the case of well-known impulse function (Dirac delta function) $\delta(\mathbf{x}), \mathbf{x} \in \mathbb{R}^{N}$, underlying problems can still be solved without formulating them using impulse functions over hypersurfaces; however, utilization of these functions paves the way for simpler descriptions and provides a better insight.

Manuscript received February 24, 2006; revised June 7, 2006. This work was supported by EC within FP6 under Grant 511568 with the acronym 3DTV. The associate editor coordinating the review of this manuscript and approving it for publication was Dr. Zhou Wang.

The author is with the Electrical and Electronics Engineering Department, Bilkent University, TR-06800 Ankara, Turkey (e-mail: onural@ bilkent.edu.tr).

Digital Object Identifier 10.1109/LSP.2006.881523
In this letter, the manifolds on which the impulse functions are defined are restricted to be hyperplanes of any dimensionality in $\mathbb{R}^{N}$. For this case, the definitions given in [1] and [2] become equivalent. Fourier transforms of such impulse functions can be found in [3] and [4]. In this paper, those Fourier transform relations are revisited using a notation consistent with the notation used in [2] and by emphasizing their importance in signal processing.

Furthermore, the well-known projection-slice theorem is revisited using the impulse functions over hyperplanes [5], [6].

Finally, the scalar optical diffraction relation between two parallel planes is derived once more using the presented projection-slice approach. This is an exact relationship based on plane-wave decomposition of the optical field [7].

We have "slices" in the space domain and "projections" in the Fourier domain; this is contrary to the common usage of the projection-slice theorem in signal processing but better fits to the formulation of the diffraction problem mentioned above. This choice is mathematically trivial since the functions and their Fourier transforms are dual.

\section{FOURIER TRANSFORMS OF IMPULSE FUNCTIONS OVER HYPERPLANES}

Let $\mathbb{R}^{N}$ be the $N$-dimensional space and $S$ be a $(N-p)$-dimensional hyperplane, in $\mathbb{R}^{N}$, passing through the origin. The impulse function $\delta_{S}(\mathbf{x})$ represents a uniform concentration (of mass) over the hyperplane $S$; please see [2] for the definitions and the notation. Simply, the Fourier transform of this function is

$$
\mathcal{F}\left\{\delta_{S}(\mathbf{x})\right\}=\int_{\mathbb{R}^{N}} \delta_{S}(\mathbf{x}) e^{-j \boldsymbol{\omega}^{T} \mathbf{x}} \mathbf{d} \mathbf{x} .
$$

Since it is rather difficult to evaluate this integral, we first find the Fourier transform of the impulse function over another hyperplane, $S_{\mathbf{R}}$, which is the rotated version of $S$, such that, for all $\mathbf{x}$ on $S, \mathbf{R x}$ is on $S_{\mathbf{R}}$, where $\mathbf{R}$ is a rotation matrix. We choose $\mathbf{R}$ so that $S_{\mathbf{R}}$ coincides with the $x_{1}=x_{2}=\cdots=x_{p}=0$ hyperplane. It is rather easy to find the Fourier transform of the impulse over $S_{\mathbf{R}}$, as

$$
\begin{aligned}
\mathcal{F} & \left\{\delta_{S_{\mathbf{R}}}(\mathbf{x})\right\}=\int_{\mathbb{R}^{N}} \delta_{S_{\mathbf{R}}}(\mathbf{x}) e^{-j \boldsymbol{\omega}^{T} \mathbf{x}} d \mathbf{x} \\
& =\int_{S_{\mathbf{R}}} e^{-j \boldsymbol{\omega}^{T} \mathbf{x}} d S \\
& =\int_{x_{p+1}, \ldots, x_{N}} e^{-j\left(\omega_{p+1} x_{p+1}+\cdots+\omega_{N} x_{N}\right)} d x_{p+1} \cdots d x_{N} \\
& =(2 \pi)^{N-p} \delta_{Q_{\mathbf{R}}}(\boldsymbol{\omega})
\end{aligned}
$$




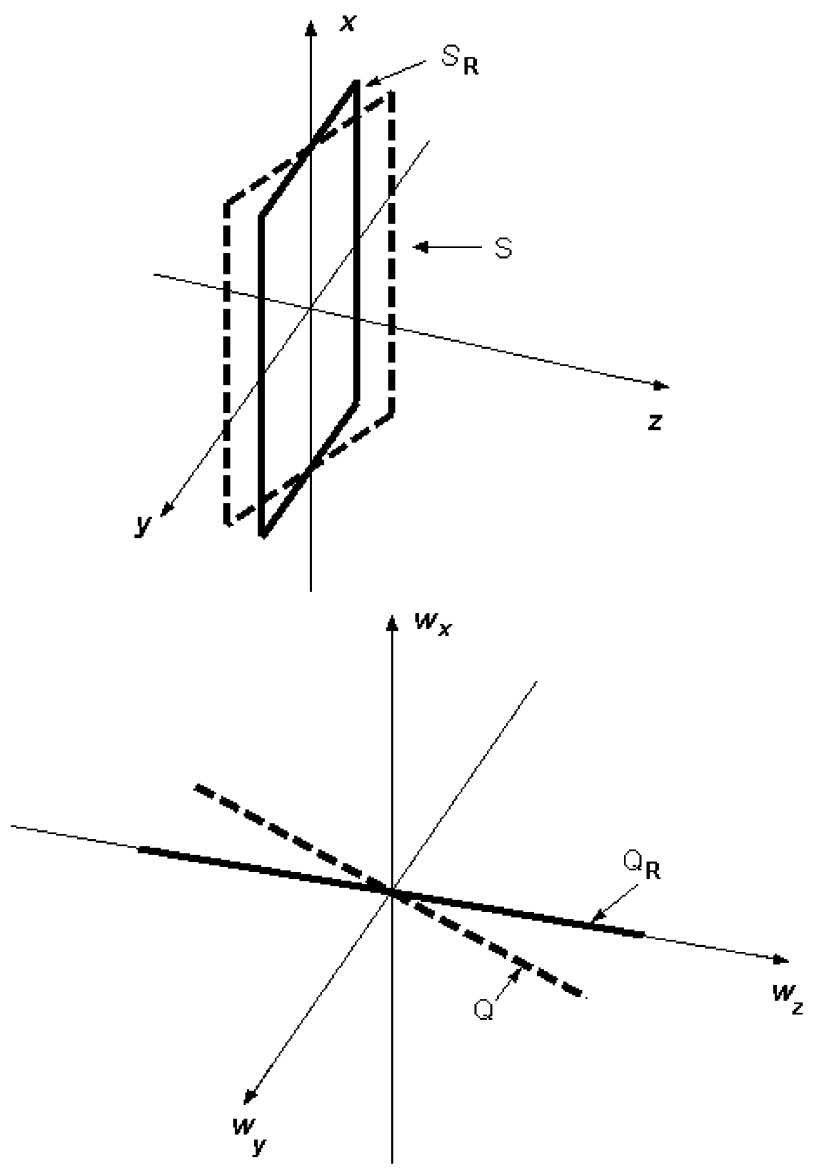

Fig. 1. Fourier transforms of impulse functions over planes. (a) Original tilted plane $S$ and the rotated plane $S_{\mathbf{R}}(z=0)$ in 3-D space. The impulse function may be loosely interpreted as a function that is "infinity" on the planes and "zero" everywhere else. (b) Fourier transforms of the planes given in (a): the Fourier transform of $\delta_{S}(\mathbf{x})$ is an impulse function over the indicated line $Q$ that is orthogonal to $S$, and the Fourier transform of $\delta_{S_{\mathbf{R}}}(\mathbf{x})$ is an impulse function over $Q_{\mathbf{R}}$ that is orthogonal to $S_{\mathbf{R}}$.

where $Q_{\mathbf{R}}$ is the $p$-dimensional orthogonal hyperplane to $S_{\mathbf{R}}$ passing through the origin. In other words, $S_{\mathbf{R}}$ and $Q_{\mathbf{R}}$ intersect orthogonally at the origin. More specifically, as a consequence of the definition of $S_{\mathbf{R}}, \delta_{Q_{\mathbf{R}}}(\mathbf{x})$ is an impulse over the hyperplane defined by $x_{p+1}=x_{p+2}=\cdots=x_{N}=0$.

An example may help to clarify the notation: let $N=3$, and let $S_{\mathbf{R}}$ be the $\left(x_{2}, x_{3}\right)$-plane, represented by $x_{1}=0$ (2-D); therefore, $p=1$. The Fourier transform of the impulse function over this plane, $\delta_{S_{\mathbf{R}}}(\mathbf{x})$, will be $(2 \pi)^{2} \delta_{Q_{\mathbf{R}}}(\boldsymbol{\omega})$, where $Q_{\mathbf{R}}$ is the line $x_{2}=x_{3}=0$ (1-D). This is shown in Fig. 1 .

Knowing that $\mathcal{F}\{f(\mathbf{R x})\}=F(\mathbf{R} \boldsymbol{\omega})$, where $F(\boldsymbol{\omega})=$ $\mathcal{F}\{f(\mathbf{x})\}$, and $\mathcal{F}\{\cdot\}$ represents the Fourier transform, we can easily find the Fourier transform of $\delta_{S}(\mathbf{x})$

$$
\begin{aligned}
\mathcal{F}\left\{\delta_{S}(\mathbf{x})\right\} & =\mathcal{F}\left\{\delta_{S_{\mathbf{R}}}(\mathbf{R x})\right\} \\
& =(2 \pi)^{N-p} \delta_{Q_{\mathbf{R}}}(\mathbf{R} \boldsymbol{\omega}) \\
& =(2 \pi)^{N-p} \delta_{Q}(\boldsymbol{\omega})
\end{aligned}
$$

where $Q$ and $Q_{\mathbf{R}}$ are hyperplanes related simply by rotation: if $\boldsymbol{\omega} \in Q$, then $\mathbf{R} \boldsymbol{\omega} \in Q_{\mathbf{R}}$. Therefore, $Q$ is the hyperplane orthogonal to $S$ and passes through the origin. An example is shown in Fig. 1.

\section{Projection-SLICE TheOREM Using IMPULSE FUNCTIONS OVER HYPERPLANES}

With the definitions and notation adopted in the previous section and in [2], the slice (in space domain) of an $N$-dimensional function, $f(\mathbf{x}), \mathbf{x} \in \mathbb{R}^{N}$, by a $(N-p)$-dimensional hyperplane $S$ in $\mathbb{R}^{N}$ is simply $f_{S}(\mathbf{x}) \triangleq f(\mathbf{x}) \delta_{S}(\mathbf{x})$. The Fourier transform, $F_{S}(\boldsymbol{\omega})$ of the slice $f_{S}(\mathbf{x})$, using well-known Fourier transform properties, is

$$
\begin{aligned}
F_{S}(\boldsymbol{\omega}) & =\mathcal{F}\left\{f_{S}(\mathbf{x})\right\}=\mathcal{F}\left\{f(\mathbf{x}) \delta_{S}(\mathbf{x})\right\} \\
& =\left(\frac{1}{2 \pi}\right)^{N}\left[F(\boldsymbol{\omega}) *(2 \pi)^{N-p} \delta_{Q}(\boldsymbol{\omega})\right] \\
& =\left(\frac{1}{2 \pi}\right)^{p} \int_{\mathbb{R}^{N}} F(\boldsymbol{\gamma}) \delta_{Q}(\boldsymbol{\omega}-\boldsymbol{\gamma}) d \boldsymbol{\gamma} \\
& =\left(\frac{1}{2 \pi}\right)^{p} \int_{Q_{\mathbf{u}}} F(\boldsymbol{\gamma}) d S
\end{aligned}
$$

where $*$ represents $N$-dimensional convolution, and $Q_{\mathbf{u}}$ is the $p$-dimensional hyperplane crossing $S$ orthogonally at a point $\mathbf{u}$ on $S ; d S$ simply means the integral is a surface integral over the indicated hyperplane. The last line in the above integral indicates that $F_{S}(\boldsymbol{\omega})$ is a "projection" in the sense of the projection-slice theorem. The last equation deserves some more comments. First of all, the last two lines imply that $\delta_{Q_{\mathrm{u}}}(\boldsymbol{\gamma})=$ $\delta_{Q}(\boldsymbol{\omega}-\gamma)$; in other words, $Q_{\mathbf{u}}$ is the shifted version of $Q$, such that if $\boldsymbol{\omega}-\boldsymbol{\gamma}$ is in $Q$, then $\boldsymbol{\gamma}$ is in $Q_{\mathbf{u}}$. Furthermore, $\delta_{Q}(\boldsymbol{\omega}-\boldsymbol{\gamma})$ is the flipped (with respect to origin) and shifted (by $\omega$ ) version of $\delta_{Q}(\gamma)$. The flip is ineffective for the integrals above since $Q$ passes through the origin. Furthermore, since we are dealing with hyperplanes (they extend to infinity), shifts of $Q$ along its own orientation will yield the same hyperplane, $Q$. Shifts along other directions will generate parallel hyperplanes to $Q$. Therefore, the value of the integral (projection) is a function of the point $\mathbf{u}$, which is the intersection of $Q_{\mathbf{u}}$ and $S$. The resultant $F_{S}(\boldsymbol{\omega})$ is, therefore, a function of $\mathbf{u}$ only: $F_{S}(\boldsymbol{\omega})=$ $F_{S}(\mathbf{u}(\boldsymbol{\omega}), \mathbf{v}(\boldsymbol{\omega})) \triangleq G_{S}(\mathbf{u}, \mathbf{v}) \triangleq G_{S}^{N-p}(\mathbf{u})$, where $\mathbf{u}(\boldsymbol{\omega})$ is in $S$. (Therefore, $\mathbf{v}(\boldsymbol{\omega})$ is the direction normal to $S$.) Therefore, $\mathbf{u}(\boldsymbol{\omega})$ is $(N-p)$-dimensional, and $\mathbf{v}(\boldsymbol{\omega})$ is $p$-dimensional. We may also choose to write, $f_{S}(\mathbf{x})=f_{S}(\mathbf{y}(\mathbf{x}), \mathbf{z}(\mathbf{x})) \triangleq g_{S}(\mathbf{y}, \mathbf{z})$, and then by restricting $\mathbf{x}$ to be on $S$, we define $g_{S}^{N-p}(\mathbf{y}) \triangleq$ $f_{S}(\mathbf{y}(\mathbf{x}))$. Therefore, as expected, we can have $(N-p)$-dimensional slice $g_{S}^{N-p}(\mathbf{y})$ and its $(N-p)$-dimensional Fourier transform, $G_{S}^{N-p}(\mathbf{u})$. (Here in the previous sentences, the superscript $(N-p)$ is used to stress that those functions with that superscript are $(N-p)$-dimensional.) Thus, we arrive to the well-known result: the Fourier transform of a slice in the space domain will be a projection in the Fourier domain. The projections of a function are called the Radon transform [6], [8].

To summarize, the slice $f_{S}(\mathbf{x})$ of $f(\mathbf{x})$ is represented as an $N$-dimensional function, $f(\mathbf{x}) \delta_{S}(\mathbf{x})$ (a multiplication), and the Fourier transform of this function, $F_{S}(\boldsymbol{\omega})$, as another $N$-dimensional function $(1 / 2 \pi)^{p} \int_{\mathbb{R}^{N}} F(\boldsymbol{\gamma}) \delta_{Q}(\boldsymbol{\omega}-\boldsymbol{\gamma}) d \boldsymbol{\gamma}$ (a convolution, as expected). This was possible by properly defining and utilizing the impulse functions over hyperplanes. Such representations may ease the utilization of these operations in signal processing: 
handling functions using well-known Fourier transform properties should be a benefit in applications by providing a better insight.

For example, using the $N$-dimensional representation of slices and their Fourier transforms via given impulse functions, we can easily find the relation for the translated slice, $f(\mathbf{x}) \delta_{S}(\mathbf{x}-\boldsymbol{\xi})$ : using simple Fourier transform relations, we know that $\mathcal{F}\left\{\delta_{S}(\mathbf{x}-\boldsymbol{\xi})\right\}=(2 \pi)^{N-p} \delta_{Q}(\boldsymbol{\omega}) e^{-j \boldsymbol{\omega}^{T} \boldsymbol{\xi}}$. Then, we can immediately write

$$
\mathcal{F}\left\{f(\mathbf{x}) \delta_{S}(\mathbf{x}-\boldsymbol{\xi})\right\}=\left(\frac{1}{2 \pi}\right)^{p} \int_{Q_{\mathbf{u}}} F(\boldsymbol{\gamma}) e^{-j \boldsymbol{\gamma}^{T} \boldsymbol{\xi}} d S .
$$

Therefore, we find that the Fourier transform of a translated slice is the projection of a modulated Fourier transform of the original function.

From the discussions above, we also know that the rotated slice $f(\mathbf{x}) \delta_{S_{\mathbf{R}}}(\mathbf{x})$ has its Fourier transform as projections onto the rotated hyperplane $S_{R}$

$$
\begin{aligned}
\mathcal{F}\left\{f(\mathbf{x}) \delta_{S_{\mathbf{R}}}(\mathbf{x})\right\} & =\left(\frac{1}{2 \pi}\right)^{p} \int_{\mathbb{R}^{N}} F(\boldsymbol{\gamma}) \delta_{Q_{\mathbf{R}}}(\boldsymbol{\omega}-\boldsymbol{\gamma}) d \boldsymbol{\gamma} \\
& =\left(\frac{1}{2 \pi}\right)^{p} \int_{Q_{\mathbf{R}, \mathbf{u}}} F(\boldsymbol{\gamma}) d S
\end{aligned}
$$

as a consequence of (5).

Combining the two properties above, we can easily get the Fourier transform of a slice by an arbitrarily oriented hyperplane that can always be represented as a rotated and translated version of a hyperplane passing through the origin: Fourier transform of rotated and translated slice, $f(\mathbf{x}) \delta_{S_{\mathbf{R}}}(\mathbf{x}-\boldsymbol{\xi})$, is

$$
\begin{aligned}
\mathcal{F}\{ & \left.f(\mathbf{x}) \delta_{S_{\mathbf{R}}}(\mathbf{x}-\boldsymbol{\xi})\right\} \\
& =\left(\frac{1}{2 \pi}\right)^{p} \int_{R^{N}} F(\boldsymbol{\gamma}) \delta_{Q_{\mathbf{R}, \boldsymbol{\xi}}}(\boldsymbol{\omega}-\boldsymbol{\gamma}) d \boldsymbol{\gamma} \\
& =\left(\frac{1}{2 \pi}\right)^{p} \int_{Q_{\mathbf{R}, \boldsymbol{\xi}, \mathbf{u}}} F(\boldsymbol{\gamma}) e^{-j \boldsymbol{\gamma}^{T} \boldsymbol{\xi}} d S
\end{aligned}
$$

which is the projection of the modulated Fourier transform of the original function, onto the rotated and translated slice $S_{\mathbf{R}, \xi}$. Here $Q_{\mathbf{R}, \xi}$ is the plane orthogonal to $S_{\mathbf{R}, \xi}$ and passing through the origin, and $Q_{\mathbf{R}, \xi, \mathbf{u}}$ is its translated version so that it crosses $S_{\mathbf{R}, \xi}$ at $\mathbf{u}$.

The simple properties outlined above will be utilized in the application given in the next section.

\section{Monochromatic ScAlar DifFraction}

It is well known that a scalar light field, $\psi(\mathbf{x})$, can be decomposed into planar waves, as

$$
\psi(\mathbf{x})=\int_{\mathbf{k}} B(\mathbf{k}) e^{j \mathbf{k}^{T} \mathbf{x}} d \mathbf{k}=\mathcal{F}_{3 D}^{-1}\left\{8 \pi^{3} B(\mathbf{k})\right\}
$$

where $\mathbf{x} \in \mathbb{R}^{3}, \mathcal{F}_{3 D}^{-1}$ represents the 3-D inverse Fourier transform, $\mathrm{k}$ is the wave-number vector representing the direction and the frequency of the propagating plane wave, and $B(\mathbf{k})$ is the amplitude of that 3-D plane wave component [2]. If the field consists only of a monochromatic light, then we have the restriction $|\mathbf{k}|=k=(2 \pi / \lambda)$, where $\lambda$ is the wavelength of the monochromatic light. We exclude any evanescent wave components and assume that the field consists only of propagating waves. Therefore, we can write $B(\mathbf{k})=\delta_{K}(\mathbf{k}) A(\mathbf{k})$, where $K$ is the sphere with radius $k$. This simple description is another demonstration of the use and power of the impulse functions over surfaces. If there are further restrictions on the direction of propagation, the surface $K$ will then be a segment (or segments) of the sphere.

In classical diffraction problems, usually the relation between diffraction patterns over planes is of interest. The simplest case is the diffraction between two parallel planes. However, given the 3-D field, a field pattern over a plane is just a 2-D "slice" in the 3-D space. It is seen in the previous section that slices can be elegantly represented by the introduced impulse functions. Therefore, the diffraction relation between planar surfaces can be easily handled using slices as follows.

Let us restrict the propagation of monochromatic light to be along the positive $z$-direction; therefore, the $k_{z}$ component of $\mathbf{k}=\left[\begin{array}{lll}k_{x} & k_{y} & k_{z}\end{array}\right]^{T}$ must be positive; and this restricts the surface $K$ to be the corresponding semi-sphere. Therefore, we can simply represent the field over a plane perpendicular to $z$-axis (i.e., $z=0$ plane) as $\psi_{0}(\mathbf{x}) \triangleq \psi(\mathbf{x}) \delta_{S_{1}}(\mathbf{x})$ (let us call $S_{1}$ the "object plane," as usually done in optics). The "diffraction plane" will be the $z=Z$ plane; let us denote it as $S_{2}$, and therefore, the field over this plane will be $\psi_{Z}(\mathbf{x}) \triangleq \psi(\mathbf{x}) \delta_{S_{2}}(\mathbf{x})$. The desired relation between these two diffraction patterns can be easily found using the projection-slice relations presented in the previous section.

The Fourier transform, $\Psi_{0}(\mathbf{k})$, of $\psi_{0}(\mathbf{x})$ is the projection given by

$$
\begin{aligned}
\Psi_{0}(\mathbf{k}) & =\mathcal{F}\left\{\psi_{0}(\mathbf{x})\right\}=\mathcal{F}\left\{\psi(\mathbf{x}) \delta_{S_{1}}(\mathbf{x})\right\} \\
& =\frac{1}{2 \pi} \int_{\mathbb{R}^{3}} \Psi(\boldsymbol{\gamma}) \delta_{Q_{1}}(\mathbf{k}-\boldsymbol{\gamma}) d \boldsymbol{\gamma} \\
& =\frac{1}{2 \pi} \int_{Q_{1, \mathbf{k}}} \Psi(\boldsymbol{\gamma}) d \boldsymbol{\gamma}
\end{aligned}
$$

which directly follows the projection property of (5). Substituting $(2 \pi)^{3} A(\mathbf{k}) \delta_{K}(\mathbf{k})$ for $\Psi(\mathbf{k})$, we get

$$
\begin{aligned}
\Psi_{0}(\mathbf{k}) & =(2 \pi)^{2} \int_{\mathbb{R}^{3}} A(\boldsymbol{\gamma}) \delta_{K}(\boldsymbol{\gamma}) \delta_{Q_{1}}(\mathbf{k}-\boldsymbol{\gamma}) d \boldsymbol{\gamma} \\
& =\frac{(2 \pi)^{2} A\left(k_{x}, k_{y}, \sqrt{k^{2}-k_{x}^{2}-k_{y}^{2}}\right)}{\cos \phi\left(k_{x}, k_{y}\right)}
\end{aligned}
$$

where we arrived at the final line by using the crossing property given in [2, (12)], and $\phi(\mathbf{k})$ is the angle between the $Q_{1, \mathbf{k}}$ (shifted $Q_{1}$ ) and the surface normal of the semi-sphere $K$ at the point of their intersection. (Please see Fig. 2.) Therefore, $\Psi_{0}(\mathbf{k})$ 


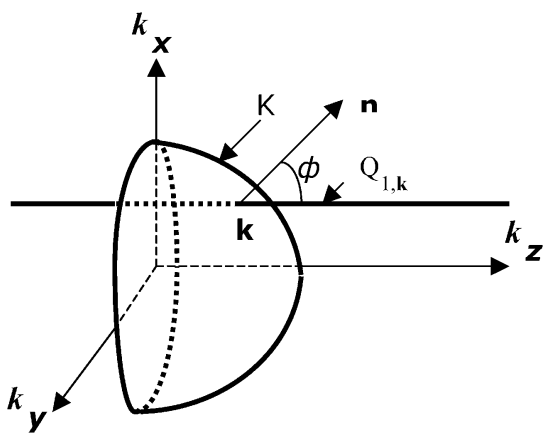

Fig. 2. Projection in the Fourier domain to describe the Raleigh-Sommerfeld diffraction due to propagating waves between two parallel planes. The spectrum of monochromatic waves propagating in the positive $z$-direction is an impulsive function over the semi-sphere $K$. The projections of this spectrum onto the $\left(k_{x}, k_{y}\right)$ plane are integrals taken along 1-D path $Q_{1, \mathrm{k}}$; the same projection can be interpreted as 3-D integrals of the multiplication of two impulsive functions: one over the semi-sphere and one over the integral line. The integration line is shifted to cover all projections of the semi-sphere.

is a 3-D function with no variation (constant) along the $k_{z}$ direction. Due to the spherical form of surface $K, \cos \phi\left(k_{x}, k_{y}\right)=$ $\left(\sqrt{k^{2}-k_{x}^{2}-k_{y}^{2}} / k\right)$. Similarly, for the diffraction plane, $S_{2}$

$$
\Psi_{Z}(\mathbf{k})=\mathcal{F}\left\{\psi_{Z}(\mathbf{x})\right\}=\mathcal{F}\left\{\psi(\mathbf{x}) \delta_{S_{2}}(\mathbf{x})\right\} .
$$

However, we also know that $S_{1}$ and $S_{2}$ are parallel, and therefore, $S_{2}$ is just a shifted version of $S_{1}$. Therefore, $\delta_{S_{2}}(\mathbf{x})=$ $\delta_{S_{1}}(\mathbf{x}-\mathbf{z})$, where $\mathbf{z}=\left[\begin{array}{lll}0 & 0 & Z\end{array}\right]^{T}$. Therefore, using the shifted slice property of (6), we know that

$$
\begin{aligned}
& \Psi_{Z}(\mathbf{k}) \\
& \quad=\mathcal{F}\left\{\psi(\mathbf{x}) \delta_{S_{1}}(\mathbf{x}-\mathbf{z})\right\} \\
& \quad=\frac{(2 \pi)^{2} A\left(k_{x}, k_{y}, \sqrt{k^{2}-k_{x}^{2}-k_{y}^{2}}\right) e^{-j \sqrt{k^{2}-k_{x}^{2}-k_{y}^{2}} Z}}{\cos \phi\left(k_{x}, k_{y}\right)} .
\end{aligned}
$$

As a final step, we note that

$$
H_{Z}(\mathbf{k}) \triangleq \frac{\Psi_{Z}(\mathbf{k})}{\Psi_{0}(\mathbf{k})}=e^{-j \sqrt{k^{2}-k_{x}^{2}-k_{y}^{2}} Z}
$$

represents the transfer function of a 3-D linear shift invariant system, which represents the change in the phase of each planewave component as we go from object plane $S_{1}$ to diffraction plane $S_{2}$, as expected. Please note that $H_{Z}(\mathbf{k})$ is a 3-D function that has no variation (constant) along the $k_{z}$ direction. It is instructive to write the same ratio (transfer function) in 2-D. Using the arguments presented in Section III regarding the relation between higher dimensional functions with no variation along some directions and their 2-D counterparts, we can write

$$
H_{Z, 2 D}\left(k_{x}, k_{y}\right) \triangleq \frac{\Psi_{Z, 2 D}\left(k_{x}, k_{y}\right)}{\Psi_{0,2 D}\left(k_{x}, k_{y}\right)}=e^{-j \sqrt{k^{2}-k_{x}^{2}-k_{y}^{2}} Z} .
$$

This is the transfer function of a 2-D linear shift invariant system that represents the exact scalar diffraction between two parallel planes due to propagating monochromatic waves.

\section{CONCLUSION}

We have shown that the impulse functions over surfaces, and the associated interpretation of the projection-slice theorem, are powerful tools to describe the fundamental mathematical nature of some well-known physical problems. For example, the exact optical diffraction relation between two parallel planes due to propagating monochromatic waves is reformulated and solved using the presented tools. Other more difficult optical diffraction relations corresponding to more complicated geometries can be solved by similar approaches using the presented tools. The basic steps for the diffraction formulation and the solution can be summarized as 1) use the 3-D functions for representing the diffraction even if we have 2-D signals (2-D patterns over planes), 2) use well-defined impulse functions over surfaces for that 2-D to 3-D transition, 3) represent the amplitude of plane wave components that superpose to make the 3-D light field as $\left.A(\mathbf{k}) \delta_{K}(\mathbf{k}), 4\right)$ represent the two parallel planes for which we sought the diffraction relation as slices of 3-D functions by 2-D planes, and finally, 5) use the developed projection-slice formulation based on impulse functions over planes.

\section{REFERENCES}

[1] I. M. Gel'fund and G. E. Shilov, Generalized Functions, Volume 1: Properties and Operations.. New York: Academic, 1968.

[2] L. Onural, "Impulse functions over curves and surfaces and their application to diffraction," J. Math. Anal. Appl., 2006, to be published.

[3] I. M. Gel'fund and G. E. Shilov, Generalized Functions, Volume 2: Spaces of Fundamental and Generalized Functions.. New York: Academic, 1968.

[4] Y. A. Brychkov and A. P. Prudnikov, Integral Trasnforms and Generalized Functions.. New York: Gordon and Breach, 1989.

[5] A. K. Jain, Fundamentals of Digital Image Processing. Englewood Cliffs, NJ: Prentice-Hall, 1989.

[6] I. M. Gel'fund, M. I. Graev, and N. Vilenkin, Generalized Functions, Volume 5: Integral Geometry and Representation Theory. New York: Academic, 1968.

[7] J. W. Goodman, Introduction to Fourier Optics, 2nd ed. New York: McGraw-Hill, 1996.

[8] S. Helgason, The Radon Transform. Boston, MA: Birkhäuser, 1980. 